\title{
Research on the Status Quo and Strengthening Method of Physical Education Teachers' Career Identity
}

\author{
WANG JIAN ${ }^{1}$, SHI LIANG $^{2}$ \\ 1.Tianjin Agricultural University, Department of Physical Education \\ 2.Tianjin zhongbei middle school, Sports Group \\ Tianjin 300384, China
}

\begin{abstract}
Teacher career identity is the teacher's concept of being a teacher as a whole, that is, the overall view of teacher's value, emotion, ability and input in the continuous activities of individual and society. It means that the individual thinks that it is valuable and meaningful, and able to find fun from the inside of profession. It is the internal motivation of the teacher's selfgrowth. It refers to both a process and a state, which determines the teacher's teaching attitude and teaching behavior.
\end{abstract}

Keywords-Physical education; Career identify; Strength and development

\section{INTRODUCTION}

The teacher's occupational survival level can be divided into three levels: the first level is the external norm level: the teacher's activities are driven by external norms and responsibilities, in a passive state, once the external requirements are loose, it may affect the teacher's Work attitude and teaching effect; the second level is responsibilitydriven level: teachers transform external requirements into self-consciousness, actively abide by norms, engage in education with the attitude of the master; the third level is the level of happiness experience: teachers have self-development awareness, The teaching activities fully demonstrate the freedom and creativity of the teacher's subject and fully reflect the value of life. It should be said that these three levels are getting higher and higher, and are getting closer to the highest level of teacher activity. As far as the current group of teachers in China is concerned, teachers who work based on their desire to earn a living account for the majority. However, the profession of teachers should never stop at the first level, because the teacher profession should not only be a means for people to make a living, but also a carrier for teachers to contribute to society and undertake missions. It is also a way to self-realize and reflect happiness in life. Modern teachers should be motivated by self-realization value while gaining and enjoying their rights and being driven by interests. They should seek the sense of meaning in life in the satisfaction of multiple values, and highlight them on the basis of responsibility, power and profit. The value of self-existence.

\section{STRUCTURE OF CAREER IDENTITY OF PHYSICAL EDUCATION TEACHERS}

In the field of physical education teacher development, the issue of teacher Career Identity has also received the attention of some scholars. However, it is regrettable to study the results of the Career Identity of physical education teachers, the research tools used or the Career Identity scales from the educational circles, or the relevant literature directly from abroad. Relevant research shows that the teaching environment factors such as classroom environment, school culture, and positive/negative leadership of leadership have an important impact on the formation of teachers' Career Identity. The Career Identity of teachers in different subjects is not the same. Teachers who teach marginal disciplines identify teachers who are significantly different from the major disciplines taught. The development of the Career Identity scale for middle school PE teachers, which is very suitable for the teaching environment and teaching mode, has a high theoretical significance and application value for promoting the in-depth research in this field. Therefore, this study uses a combination of theoretical analysis and empirical testing. Firstly, theoretical analysis is used to theoretically conceive the structural dimensions of the physical education teachers' Career Identity in secondary schools, and then they are tested and corrected by empirical methods, using qualitative and quantitative combination. The research strategy explores the structure of Career Identity of middle school PE teachers in China.

\section{CHARACTERISTICS OF PHYSICAL EDUCATION TEACHERS' CAREER IDENTITY}

Career Identity is the result of the interaction between the individual experience of teachers and the social, cultural and institutional environment in which they live. To explore the characteristics of gender, teaching age, professional title and academic qualifications of middle school PE teachers' Career Identity, to comprehensively grasp the current situation and development trend of middle school PE teachers' Career Identity, and to understand more deeply the middle school PE teachers' professional psychology and targeted exploration. Effective measures to promote the Career Identity of physical education teachers are of great significance. In the related 
research at home and abroad, the characteristics of different individual characteristics (age, gender, professional title, education, teaching subjects, etc.) and teachers' Career Identity from different environments (school level, location, etc.) have been studied. Because the teachers in these studies are from different groups, the characteristics of Career Identity are also very different. This study will explore the characteristics of Career Identity of physical education teachers in middle schools.

The higher overall Career Identity of middle school PE teachers is consistent with the overall development of China's education. On the one hand, in recent years, education has been placed in a priority position. The treatment of teachers in China, especially in the basic education stage, has been greatly improved. The state has invested more and more in teacher education, and the social status of teachers has gradually improved. Improvement, the improvement of the external environment has an important impact on the improvement of teachers' Career Identity; on the other hand, the decline of the physique and health level of Chinese students has made the school sports work pay more attention and pay more attention to the development of physical education teachers. . In the factors of teacher Career Identity, except for the continuous identity factor, the average value is greater than 4 , which is in the upper level. The average value of each factor is: value identification $>$ input identification $>$ ability identification $>$ emotional identification $>$ continuous identification. The high school physical education teachers score the highest on the value identification factor. The reasons may be: 1 The important role of teachers in social development and human progress has become a social consensus; 2 With the gradual advancement of quality education in China, the status of physical education in the overall development of students' education More and more attention is paid to, and the majority of physical education teachers have gained more understanding of the professional value of physical education teachers by learning the concept of new curriculum reform and participating in continuing education activities.

Middle school physical education teachers have the lowest scores on the continuous identity factor. The reasons may be: 1 In many middle schools, sports work has not attracted the attention of decision makers. In the interviews, we found that middle school physical education teachers "different pay for equal work" and lack of job promotion opportunities. There are many cases of insufficient equipment, which affects the determination to continue to engage in physical education; 2 physical education teachers are mostly attending classes outdoors, and they are exposed to the sun all the year round. The burden of the body is heavier, so some teachers have sought to go to school. Opportunities for the work of the organs and education authorities; 3 Many teachers do not like sports before they join the job. Many of them are the contents of the professional examinations before the college entrance examination, and they enter the college sports majors with the advantage of the cultural course. For them, they are sports. After the teacher, if the fun of the physical education teacher cannot be found in the teaching practice, it is difficult to maintain the enthusiasm for the physical education teacher profession.
In the mature period of career (teaching age 16-25, age 4049), the physical education teachers in the middle school achieved the highest ability identification factor, but did not significantly improve the Career Identity and other four factors, even in the identification, emotion The mean value of the factors such as identity shows a trough. The reason may be: First, the teachers at this stage have been engaged in teaching for about 20 years, accumulated rich teaching experience, formed their own teaching characteristics, and their ability identification reached a high level. Because of this, they are very easy to enter the "plateau period" of teaching career, and believe that "even if you invest less, you can get the same teaching effect" 2; Second, the physical education teachers at this stage will be more stable in terms of titles and incomes. The honors obtained are also the most, and when these goals are realized, the space for further development is not large, and there may be doubts about the value and meaning of their own professions, and there is no more positive experience for the work. I also got it, I feel that there is nothing exciting. "Sports teachers are not a physical education teacher." Third, according to the normal situation, the teachers at this stage are in the most brilliant period of their lives, but also the economy and life. When the pressure is greatest, the family aspect is "old and small," and the increase in physical and psychological stress and work intensity has greatly increased the chances of starting occupational diseases. In addition, the income and social status of secondary school physical education teachers are not Too high, if you can't find the meaning and value of physical education teachers' work, it is easy to be in the same age as your peers. Loss generated in the mood, and produce Career Identity recession levels. Therefore special attention should be paid to the physical education teachers in the teaching age group. Beijaard (1995) found that at the beginning of his career, teachers' Career Identity is relatively low; as the number of years of service increases, teachers' perception of Career Identity tends to be positive. The results presented in this study are quite different, and even show a downward trend in the two factors of value identification and emotional identity. The reasons for these problems may be: 1 The environment for the work of physical education teachers in middle schools needs to be improved. In the interviews, we found that sports work is not paid enough attention in the school. The investment in venues and equipment is not enough. The physical education teachers are promoted in the title. And the performance evaluation often fails to obtain the same policy as the "main subject"; 2 the reflective ability of the majority of physical education teachers needs to be strengthened, and while the ability is gradually improved, the meaning and value of physical education teachers should be deepened, because teachers are active. Career Identity can also help them overcome their dissatisfaction with poor working conditions, weaken teachers' turnover intentions, and gain more external support for the development of sports work through good work performance and teaching effects. 


\section{THE FORMATION PROCESS OF TEACHERS' CAREER IDENTITY}

Teachers' outlook on their future career is an important connotation of teachers' Career Identity. The prospect of middle school PE teachers' career prospects, that is, the pursuit of continuing work is an important manifestation of continuous identification and input recognition in Career Identity. Teacher Zhao did not work long hours, but determined to be a physical education teacher. Facing the reality of confusion, he chose to be a good class teacher first, proving that "sports teachers can also be good class teachers, no worse than others", and then improve the physical education teachers. In addition to continuing to improve his business level, Mr. Liu discovered some psychological problems of students through physical education classes and class teacher experiences. He participated in the training of psychological counselors at his own expense and conducted psychological counseling for students. In his narrative, $\mathrm{Mr}$. Wang The second time emphasized that "I like to go to class, I like to lead the team". I don't want to influence his investment in students because of too many administrative matters. Sun has retired, but the teaching supervision as a sports group is still in C. High school physical education work and silent dedication. He has been cultivating young people and hopes that they will continue to deepen their physical education reforms that he has been responsible for more than two decades. Although he is older, his legs and feet are not as good as before, but he repeatedly said "I still want to continue class!". Through the above analysis, we found that all the teachers did not regard teaching sports as a means of earning a living, but inclined to think that physical education teachers are occupations that require physical and mental input. Physical education teachers will have great development for students. influences. It is precisely because these teachers have a high recognition of the profession that they have some concerns about the future development. Teacher Liu believes that gender is not a problem for physical education teachers, but they are worried that they will be older in the future. "Would you not have energy before?" "Children don't like me" and can't work as hard as they do now. Teacher Wang believes that after becoming a senior teacher, I feel that the challenge in my work is greatly reduced. I have some honors. If I work harder in the future, is it just "a good middle school physical education teacher?" We can't predict the future performance of these teachers, but through their fears, they have a certain degree of motivation in their current career. It may be that there is no correct reflection when the Career Identity fluctuates at a certain stage. In addition to contributing to the professional development of teachers, expanding the Career Identity and responsibility of teachers can also promote teachers to remain in their original positions. As far as our reality is concerned, we not only need to create better external conditions for the development of physical education teachers, but also promote teachers to create new school culture, increase incentives in their work, and enable every physical education teacher to update teachers from time to time. The meaning of updating the understanding of the meaning of continuing as a physical education teacher.
There are many research theories about teachers' Career Identity at home and abroad. This study is more about "discovering the teacher's subject" in epistemology and methodology, that is, starting from the teacher as "individual" and presenting each teacher according to the teacher's selfnarration. The same life world to explore the connotation and construction process of teachers' Career Identity at different stages of development. In the analysis of the four cases, we saw their different life worlds, different development environments, and their differences in the value of physical education teachers, the understanding of the relationship between teachers and students, and the attitude towards the development environment. Teacher Career Identity theme. For example, Mr. Zhao hopes to change his marginalization status in middle school through the professional advantages of physical education teachers and actively explore the working methods that do not violate his Career Identity and adapt to school culture. Teacher Liu believes that students are the meaning of her work. Constantly challenge themselves in the profession, firmly believe that Career Identity has nothing to do with teaching subjects and gender; Mr. Wang pays attention to the teacher-student interaction in teaching and the continuous investment of teachers, and strives to achieve the common development of teachers and students, and can rationally look at the existence of work. Realistic problems; Sun emphasizes the teaching reflection from the teaching practice, pays attention to the lifelong learning and the construction of the teaching team, in order to achieve his professional emotions closely related to the needs of the motherland.

Looking at these rich and personalized Career Identity themes, we can find that the Career Identity of middle school physical education teachers is not a stable teacher professional role norm, there is no established scope and content of identification, and there is no default role or goal towards an external environment. . In other words, this study does not accept the "role theory" in which the Career Identity bias is determined by the structure and the role is consistent. We believe that the construction of Career Identity of physical education teachers has no established direction and content. It is constructed from the teacher's subject and its life world, from the teacher's work and life experience, with its own language, its own reflection, and its own way. Explain your Career Identity. Since the construction of Career Identity of physical education teachers does not have clear established directions and goals, they can respond to the current national situation and educational policies, meet the actual needs of their teachers' work, or be influenced by important events and characters in their life world, so their Career Identity The content may not be exactly the same as the requirements of the external policy norms, and sometimes even conflicting and deviating. Through the bold measures of teaching reflection and teaching reform, Teacher Sun and Mr. Wang realized the organic combination of personal development goals and national policy trends. Mr. Liu's own development motivation and sense of accomplishment are high, but she has not been able to truly integrate relevant policies and measures into her Career Identity. Teacher Zhao feels the great contrast between the external environment and the internal identity, and is in the predicament of Career Identity. 


\section{CONCLUSION}

On the whole, the construction of Career Identity of middle school PE teachers is not completely restricted by the society's expectations of teachers, education policies and reforms, or the presupposition of expert authority on the role of teachers. It is not a loyal role adoption process, although these external factors It has sufficient influence on the construction of Career Identity, but the Career Identity of teachers is mixed with many personal values, beliefs, emotional abilities and situations, which are more colorful and have obvious personal characteristics. That is to say, the Career Identity of teachers is constructed from the subjective life world, and is not the essential role adopted.

\section{STRATEGY TO STRENGTHEN PHYSICAL EDUCATION CAREER IDENTITY}

First of all, it is necessary to improve the social status of the junior high school physical education teachers and the economic status, and enhance their professional self-esteem and self-improvement. Appropriately improving the treatment of junior high school PE teachers, through publicity and social work in the social work, the social atmosphere of the teacher and the teacher's professionalism, and the improvement of the teacher's professional self-esteem and self-improvement are important means to enhance their professional recognition. Second, establish an equal, reasonable, and effective incentive mechanism. To improve the Career Identity of junior high school PE teachers, in addition to improving social status and economic treatment from the social level, it is necessary to establish an equal and effective incentive mechanism in schools. Furthermore, creating a harmonious and respectful campus atmosphere enhances the sense of belonging of junior high school PE teachers. Junior high school sports teachers must also establish a correct outlook on human life and value. As a junior high school physical education teacher, it should be used as a model to overcome the difficulties in the work, actively resist the temptation of various social kinds and the impact of the "money supremacy" concept of the market economy, and establish a lofty outlook on life and values for the promotion of sustainable human development. Constantly improve their sense of Career Identity and serve the development of education in China.
The construction of physical education teachers' Career Identity is based on the subject life world, and is not the essential role. The career of physical education teachers is also how they strive to "live themselves" and "describe themselves". Teachers in different fields and at different stages are concerned about the question "Who am I?", "How do I become a physical education teacher?" "What kind of physical education teacher am I?" "Facing the school administration and teaching reform What should I do?" "What is my future?" Shows its rich Career Identity. The source of Career Identity of physical education teachers is very wide. The characteristics of teachers, the experience of students, the experience of teachers' pre-service education, the reflection in teaching practice, the experience of life development, and the perception of teaching environment are all important sources of Career Identity. . Diversified sources of identity have caused differences in the Career Identity of physical education teachers, and even conflicts of Career Identity.

\section{ACKNOWLEDGMENT}

2018 Tianjin Education committee Scientific research projects.

\section{REFERENCES}

[1] Wei Shuhua, Song Guangwen. A review of the research on the professional qualifications of foreign teachers[J]. Comparative Education Research, 2005(5): 61 64 .

[2] Song Guangwen, Wei Shuhua. Analysis of related factors in the recognition of teachers' professional careers $[\mathrm{J}]$. Psychological Development and Education, 2006(1): 80 86.

[3] Jiang Yong, et al. Research on the influence of the teacher's work satisfaction degree model [J]. Management Science, 2006 (1): 162 .

[4] Sun Meihong, Qian Qinzhen. A review of the teacher's professional recognition research [J]. Childhood Education (Teaching Science Edition), 2007 (7, 8): $59 \sim 62$.

[5] Li Jiangxia. The Introduction of Foreign Teachers' Burnout Theory to China [J]. Education Science, 2003, 19(1): 62 6 4 . 